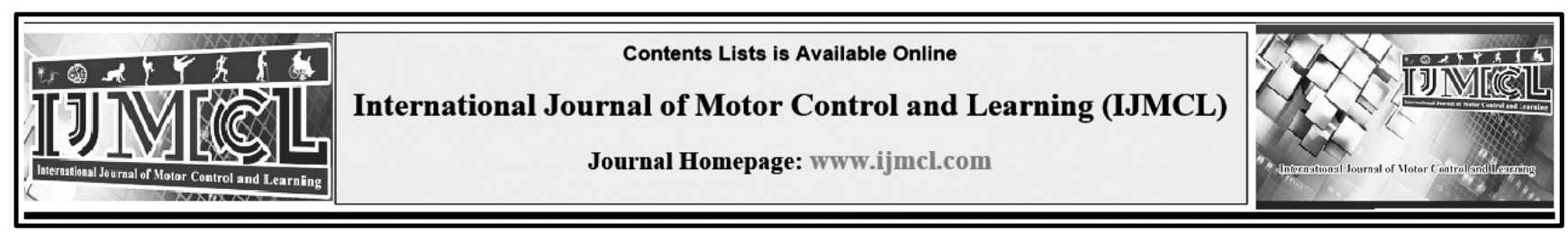

\title{
Age-related Reductions in Manual Asymmetry with Increased Visuospatial Demands
}

\author{
Tania S. Flink $\mathrm{a}^{*}$, Jose A. Matos ${ }^{\mathrm{b}}$
}

a Ph.D. Exercise Science, Department of Applied Exercise Science, Gannon University, 105 Commercial Center Drive, Ruskin, FL 33573 USA.

${ }^{\mathrm{b}}$ Master of Athletic Training (MAT), Department of Athletic Training, Gannon University, 109 University Drive, Erie, PA 16541 USA.

\begin{tabular}{|c|c|}
\hline Keywords & Abstract \\
\hline Aging & Background: Many factors influence manual asymmetry during goal-directed motor tasks, \\
\hline Manual Asymmetry & $\begin{array}{l}\text { but it is unclear how the manipulation of visuospatial information influences laterality in } \\
\text { hand control in aging adults. }\end{array}$ \\
\hline Laterality & Objective: To examine age-related changes in manual asymmetry with increased \\
\hline Visuospatial & $\begin{array}{l}\text { visuospatial processing demands. } \\
\text { Methods: Twenty young }(M=20.8, S D=3 \text { years) and } 20 \text { older }(M=69.9, S D=6 \text { years) } \\
\text { right-handed adults participated in a motor task which required pieces to be inserted into } \\
\text { spatially mapped holes (Task } 1) \text {, and a motor plus visuospatial task (Task } 2) \text {, where the } \\
\text { pieces to be inserted were not spatially mapped with the holes. Manual asymmetry was }\end{array}$ \\
\hline $\begin{array}{l}\text { Tania S. Flink, } \\
\text { Email: flink001@gannon.edu }\end{array}$ & $\begin{array}{l}\text { determined using the laterality quotient (LQ), which was computed using time to } \\
\text { completion. }\end{array}$ \\
\hline & $\begin{array}{l}\text { Results: Dominant right-hand performance was observed for Task } 1 \text { for young adults (LQ } \\
=3.91 \text { ) and older adults }(\mathrm{LQ}=5.14) \text {, but for Task } 2 \text { a more balanced performance between } \\
\text { the hands was found for older adults }(\mathrm{LQ}=-0.73 \text { ) versus young adults }(\mathrm{LQ}=6.53 \text { ), } p<\end{array}$ \\
\hline Received: $2021 / 04 / 23$ & $\begin{array}{l}0.05 \text {. } \\
\text {. }\end{array}$ \\
\hline $\begin{array}{l}\text { Accepted: } 2021 / 07 / 09 \\
\text { Published: } 2021 / 08 / 31\end{array}$ & $\begin{array}{l}\text { Conclusions: The reduction in manual asymmetry in Task } 2 \text { with age is most likely due to } \\
\text { the added demands from increased visuospatial processing, which differentially influenced } \\
\text { movement control in older compared to young adults. }\end{array}$ \\
\hline
\end{tabular}

\section{Introduction}

According to the hemispheric asymmetry reduction in older adults (HAROLD) model, neural activity in the brain is less lateralized in older adults compared to young adults (Cabeza, 2002; Cabeza, Anderson, Locantore, \& McIntosh, 2002; Dolcos, Rice \& Cabeza, 2002). As such, activity in the prefrontal cortex in young adults was found to be highly specialized for the left or right hemispheres for encoding or retrieval of memory for cognitive tests of working memory (Li, Moore, Tyner, \& Hu, 2009; Piefke, Onur, \& Fink, 2012), visuospatial working memory (Bopp \& Verhaeghen, 2007;
Cansino, Guzzon, Martinelli, Barollo, \& Casco, 2011; Hale et al., 2011), and episodic memory and retrieval (Cabeza et al., 1997). In older adults, reduced asymmetry and increased bilateral activation in the prefrontal areas was observed, particularly during recall on memory tasks. These changes were also coupled with performance changes, such as slower processing speeds for retrieval tasks and declines in response accuracy (Cabeza et al., 2004; Li et al., 2009; Park et al., 2002). According to the HAROLD model, the increased bilateral activation patterns occur in 
compensation for the age-related changes in processing of information.

For motor tasks, the influence of aging on hemispheric lateralization on performance is met with mixed results. Studies that align with the HAROLD model have shown that a reduction in manual asymmetry of performance occurs in righthanded older adults, which manifests itself in more symmetric control of the right and left hands during goal-directed motor tasks. These studies include those involving pointing to virtual targets (Przybyla, Haaland, Bagesteiro, \& Sainburg, 2011), adaptation to visuomotor perturbations (Wang, Przybyla, Wuebennhorst, Haaland, \& Sainburg, 2011), steadiness and aiming tasks (Kalisch, Wilimzig, Kleibel, Tegenthoff, \& Dinse, 2006), index and thumb opposition tapping tasks (Naccarato et al., 2006), wrist and finger extension movements (Hutchinson et al., 2002), and the Purdue pegboard test (Francis \& Spirduso, 2000). Conversely, maintenance of superior right-hand performance in right-handed older adults was observed in simple tapping and pegboard tests (Mitrushina, Fogel, D’Elia, Uchiyama, \& Satz, 1995), cued pointing tasks to targets (Chua, Pollock, Elliott, Swanson, \& Carnahan, 1995), and line tracing tasks (Francis \& Spirduso, 2000). For studies in support of the HAROLD model, the reductions in asymmetry in motor tasks are thought to be due to compensatory actions of the brain in lieu of cognitive (Naccarato et al., 2006; Przybyla et al., 2011) or peripheral (Kalisch et al., 2006) declines with age. However, preserved right-hand laterality in motor tasks in the research by Mitrushina and Chua suggests a preservation in dominant-hand performance due to movement strategy and years of practice using the right hand (Chua et al., 1995; Mitrushina et al., 1995).

More recently, Flink and Iorio (2015) examined changes in manual asymmetry from the second to the eighth decades of life using the Purdue pegboard protocol. While the older individuals inserted a significantly smaller number of pegs into the holes in the allotted time compared with the younger individuals, performance was lateralized for superior performance using the right hand for all age groups (Flink \& Iorio, 2015). The maintained manual asymmetry for right-hand performance across the decades was attributed to the simplistic nature of the Purdue pegboard test; as such, it was not complex enough to induce reductions in manual asymmetry with age (Flink \& Iorio, 2015). The mixed findings in the literature regarding reductions in manual asymmetry in older adults for motor tasks may therefore stem from the specific requirements (e.g., sensorimotor) of the task itself, which is further impacted by task complexity (Guzzetti \& Daini, 2014; Hausmann, Kirk, \& Corballis, 2004; Provins, 1997; Teixeira, 2008). As task requirements change, a person may change his or her movement strategy to meet these demands for a successful completion of a more difficult task. In some instances, a reduction in manual asymmetry may be observed, which manifests itself in a more balance performance between the hands.

For this study, changes in manual asymmetry with age were examined by specifically isolating one aspect of motor control, namely visuospatial processing. This was examined during a simple 
motor task and comparing it to a more complex task with increased visuospatial processing. It was hypothesized that the addition of the visuospatial component would tax the central nervous system in older adults such that a more symmetric control of the limbs would be observed.

\section{Method}

\section{Participants}

For this study, 20 young adults $(M=20.8, S D=3$ years) and 20 older adults $(M=69.6, S D=6$ years $)$ participated. Of the young adults, 11 were men and 9 were women. Of the older adults, 6 were men and 14 were women. Participants were recruited from a private university and from a senior center located near the university. Data were collected at both locations. Self-reported hand preference was assessed using the Edinburgh Handedness Inventory (Oldfield, 1971). The computed laterality quotients for participants from the inventory ranged from $0.7-1.0$, indicating righthand to strong right-hand preference for all participants tested. In addition, participants were screened for ambiguous hand switching (left to right hand usage) that may have occurred at a young age. The participants reported normal or corrected vision and hearing and no physical and neurological impairments that would impede performance on the tasks. All participants performed the Mini-mental State Examination and passed with a score of 27 or greater out of 30 points. This study was approved by the university Institutional Review Board, and all participants signed an approved consent form prior to testing.

\section{Equipment}

For this experiment, the game of Perfection (C) Hasbro, 2008) was used. The game consisted of a $0.16 \times 0.165 \mathrm{~m}$ platform with 25 recessed holes cut out into 25 different shapes (star, oval, X, diamond, etc.), with separate plastic pieces that corresponded with each of the shaped holes. This piece of equipment was chosen to mimic the goal-directed movements of the Purdue pegboard test (inserting small pieces into holes), while at the same time increase the complexity of the task by requiring participants to recognize shapes and scan the board for proper piece insertion. The board and its components were transported between testing locations (university and senior center).

\section{Task 1 Protocol}

Task 1 (Motor Task) was performed to examine manual asymmetry in a task with minimal visuospatial demands. Participants were seated in front of the board, which was placed at his or her midline. For this task, the shaped pieces were placed on either the right or left of the table next to the game board (for the right or left hands, respectively). These pieces were spatially mapped with the exact corresponding hole on the board (see Figure 1, Panel A_1 for left hand set-up and Panel A_2 for right-hand set-up).

Prior to data collection, participants were allowed to handle the pieces and insert a few pieces into the board for familiarization. At the beginning of each trial, participants were required to keep both hands on his or her lap, and the non-testing hand was to remain on the lap during the duration of the trial. On an auditory go signal provided by the 
researchers, participants picked up one piece at a time with the testing hand (right or left) and placed it in its corresponding hole as fast as possible. The trial terminated when all 25 pieces were inserted into their proper locations. The time to complete the trial (from the start signal to the last piece) was recorded using a stopwatch. Hand order was counterbalanced among participants. Two complete trials for each hand were performed. No specific directions were given to the participants about insertion direction (right to left, or up to down), though all participants chose to insert the pieces in a horizontal row fashion.

\section{Task 2 Protocol}

The purpose of Task 2 (Visuospatial plus Motor Task) was to examine changes in manual asymmetry with increased visuospatial processing demands. To implement this, the shaped pieces were randomly mixed together and placed in a pile to the right or the left side of the board (for rightor left-hand use, respectively, see Figure 1, Panel B_1 and Panel B_2). For this task, the participants picked up one piece at a time from the pile on an auditory go signal and placed the piece in its proper hole as fast as possible with the testing hand (right or left hand). The participants were free to choose the piece in which to insert for each movement, but they were instructed to play the piece in hand and could not choose another piece to play until the previous piece was inserted. Again, the non-testing hand was to remain on the participant's lap for the duration of the trial. The trial terminated when all 25 pieces were inserted, and the time to insert all pieces from the beginning of the trial until the final piece was inserted was recorded using a stopwatch. Two trials for each hand (right and left) were performed, and the hand order was counterbalanced among participants.

\section{Data Analysis}

For Tasks 1 and 2, the time to complete the task was recorded for each trial. Completion time was chosen over other measures (e.g., the number of pieces inserted in a designated time) because the researchers desired for 25 movements be performed for all individuals. Completion time for the two trials collected for each separate hand was averaged together so that only one value was analyzed. Completion time for the right and left hands was then used to compute the laterality quotient (LQ) using the equation below:

$$
\mathrm{LQ}=\frac{\mathrm{L}-\mathrm{R}}{\mathrm{L}+\mathrm{R}} \times 100
$$

In Equation 1, $\mathrm{L}$ and $\mathrm{R}$ corresponded to the average completion time of the left and right hands, respectively. LQ is commonly used as a measure of manual asymmetry between the hands by determining the contribution of the right versus the left hand on performance for a variety of variables. For this study, a positive LQ indicated better (or faster) performance for the right hand, while a negative LQ indicated better (or faster) performance for the left hand. A value nearing 0.0 signified a more symmetric performance between the hands.

\section{Statistical Analysis}

A 2 (Task: Tasks 1 and 2) x 2 (Group: young and older adults) analysis of variance (ANOVA) was 
used to determine differences between tasks and groups for LQ, where $\alpha=0.05$, and $p<0.05$ was considered statistically significant. For significant interactions, Tukey HSD post-hoc tests were used to examine the significant effects of the variables in the interaction. All statistics were performed using IBM SPSS statistical software.

\section{Results}

A significant main effect of Task was found for LQ, $F(1,76)=4.72, p<0.05, \eta^{2}=0.059$. LQ was significantly more positive for Task $1(M=5.23$, $S D=5)$ compared to Task $2(M=2.21, S D=7)$, indicating a more lateralized or faster performance for the right hand for Task 1 performance compared with Task 2. There was no significant main effect of Group for LQ.

A significant Task $x$ Group interaction was also found for LQ, $F(1,76)=9.42, p<0.05, \eta^{2}=0.110$, see Figure 2. Tukey HSD post-hoc analyses revealed that LQ did not significantly differ between young and older adults for Task 1 . However, LQ significantly differed between young and older adults for Task 2. LQ was significantly more positive in value for young adults $(M=5.14$, $S D=6.5)$ compared to older adults $(M=-0.73, S D$ =7.1), indicating superior (faster) right hand performance in the younger group versus the older group. Post-hoc analysis also revealed LQ did not differ for Task 1 and Task 2 for young adults. In contrast, LQ significantly decreased from a more positive value $(M=6.53, S D=7.2)$ to a less positive value nearing $0.0(M=-0.73, S D=7.1)$ from Task 1 to Task 2 in older adults, indicating a reduction in manual asymmetry with age and more balanced performance between the hands.
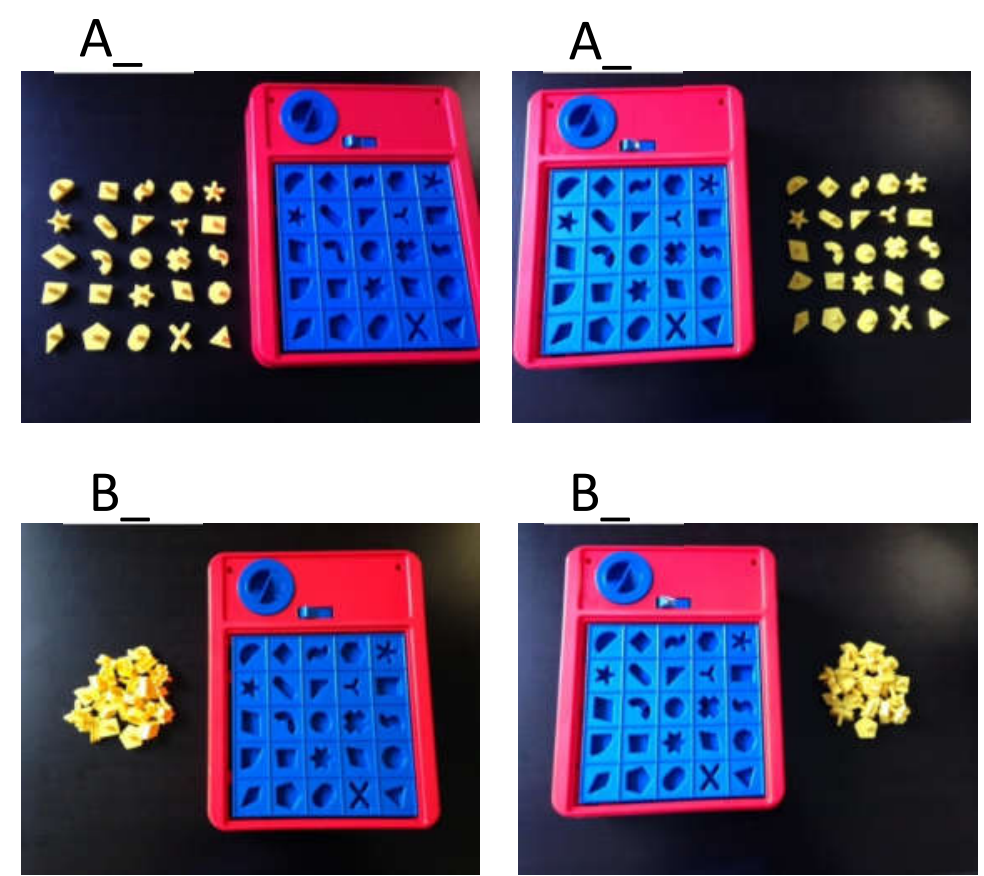

Figure 1. Experimental set-up for Task 1 (Motor Task) and Task 2 (Visuospatial plus Motor Task). A_1 and A_2 indicate the set-up for the left and right hands, respectively, for Task 1. B_1 and B_2 indicate the set-up for the left and right hands, respectively, for Task 2. 


\section{Laterality Quotient (LQ)}

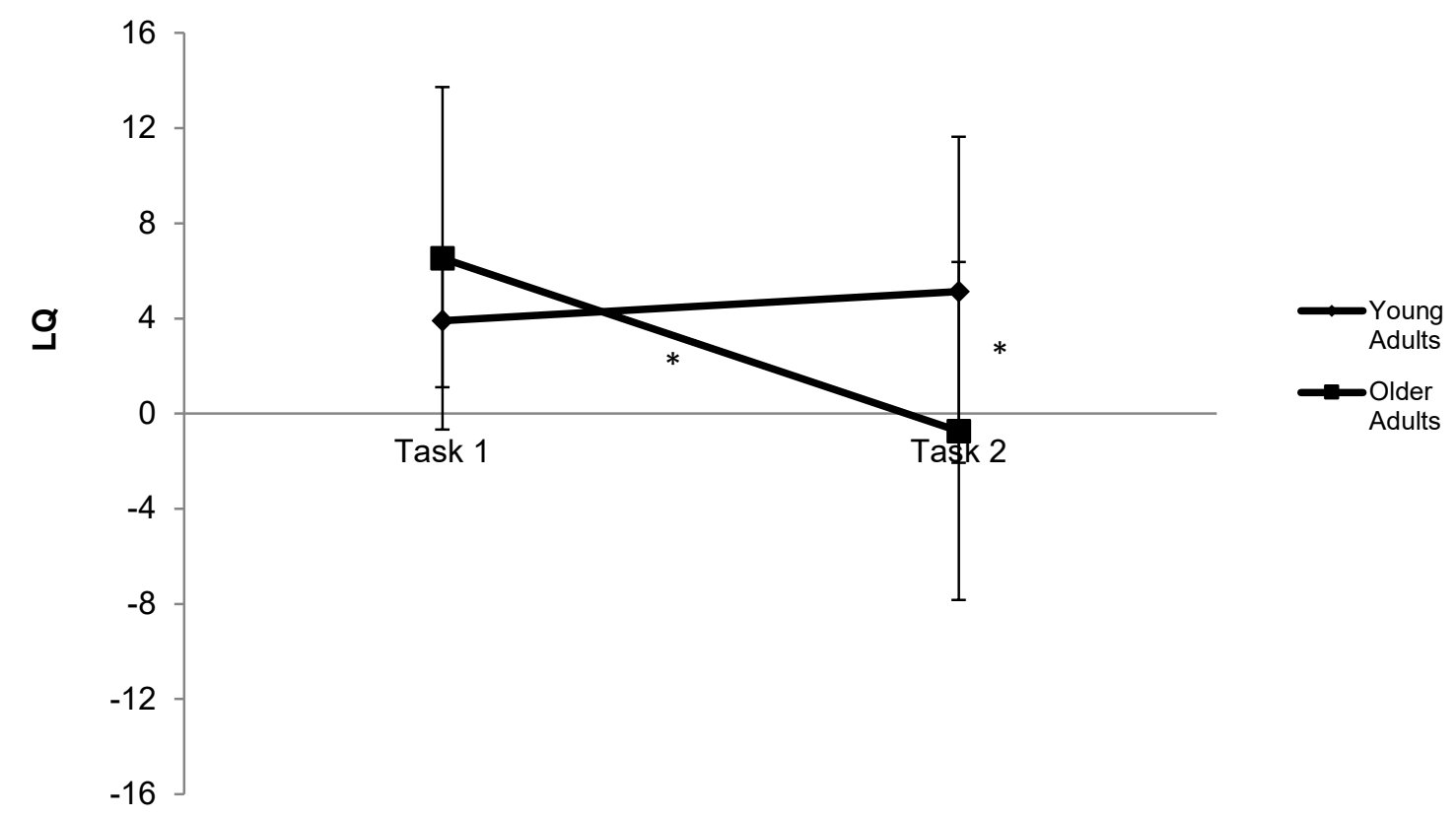

Figure 2. Computed Laterality Quotient (LQ) for young and older adults for Task 1 and 2. * indicates significant comparison.

\section{Discussion and Conclusion}

This study examined age-related changes in manual asymmetry by increasing visuospatial demands during a motor task. It was predicted that increased visuospatial demands in Task 2 compared with Task 1 would result in reductions in manual asymmetry and thus more symmetric hand movements in older adults. This hypothesis was supported by the statistical findings. Based upon the post-hoc analyses, the reduction in manual asymmetry from Task 1 to Task 2 was driven by performance in the older adult group, as LQ became significantly less positive toward a more balanced performance between the hands, while young adults maintained manual asymmetry for superior right-hand performance.
The purpose of Task 1 was to examine manual asymmetries in a situation that allowed the pieces on the table to be spatially mapped with the holes on the board, similar to the movements required for the Purdue pegboard task (Flink \& Iorio, 2015; Francis \& Spirduso, 2000). For this task, the maintenance of manual asymmetry for superior right-hand performance was observed in both young and older adults. It is suggested that the complexity of this task was not sufficient enough to produce alterations in movement control in either group. Thus, a strong advantage for use of the preferred, practiced hand may occur in simple tasks, particularly in older adults (Flink \& Iorio, 2015; Hausmann, Kirk, \& Corballis, 2004). On the other hand, a significant reduction in manual asymmetry was observed in older adults for Task 
2. The elimination visual mapping of the pieces with the board in Task 2 most likely led to greater used of visuospatial processing demands; however, in older adults, this also led to the reduction of manual asymmetry compared with Task 1 , which was not observed in the younger group.

These results from Task 2 can be interpreted a number of ways. In support of the HAROLD model, the reduction in manual asymmetry for older adults in Task 2 most likely stemmed from declines in hemispheric activation with age (Cabeza, 2002; Cabeza et al., 2002). Specifically, these declines would occur in areas such as the motor cortex for movement planning or possibly the parietal and/or temporal cortices for visual location or mapping, respectively. Declines in function in one cortical area may have resulted in recruitment of a wider area of cortical networks across both hemispheres in compensation, which may have resulted in the symmetric hand control in older adults for a motor task involving the right and left hands.

Reductions in manual asymmetry from Task 1 to Task 2 in older adults may also be due to hemispheric specialization of function when using the dominant versus the non-dominant hand. According to research by Sainburg and Wang, the dominant hand, or the dominant hemisphere, contributes to the organization of the dynamic aspects of movement (including hand path trajectory, interlimb coordination, and force control), while the non-dominant hand, or nondominant hemisphere, contributes to control of limb posture and feedback-mediated control (Sainburg, 2005; Sainburg \& Kalikanis, 2000;
Wang, D’Amato, Bambrough, Swartz, \& Miller, 2016; Wang \& Sainburg, 2007; Wang, et al., 2011). For Task 1, successful placement of the pieces was very much dependent upon proper hand path trajectory and limb coordination, hence the maintained manual asymmetry for right-hand superior control in both groups. However, for Task 2 , the reduction in manual asymmetry in older adults may have resulted from an allocation of resources shifting to the non-dominant hemisphere, which included greater feedback-mediated control and more emphasis on limb posture in lieu of the greater visuospatial processing demands. As a result, a more symmetric performance between the hands was observed due to the increased hemispheric processing within the non-dominant hemisphere.

Finally, the reduction in manual asymmetry from Task 1 to Task 2 in older adults may be a consequence of changes in sensorimotor integration with age. According to the literature, goal-directed movements in older adults are commonly performed with slower movement speeds, reduced end-point accuracy, and increased number of corrective movements compared to young adults (Baweja et al., 2015; Pohl, Winstein, \& Fisher, 1996; Seidler-Dobrin \& Stelmach, 1998; Temprado et al., 2013; Yan, Thomas, \& Stelmach, 1998; Yan, Thomas, Stelmach, \& Thomas, 2000; Yeh, Cinelli, Lyons, \& Lee, 2015). These motor changes with age were attributed to increased reliance on feedback, which affected the subjects' ability to program the movement for execution. In Task 2, a number of sensory components were required, such as greater use visual feedback 
compared with Task 1 (to scan the board for locations, to recognize shapes and patterns), proprioception (appropriate placement of hand with respect to the board), and tactile feedback (to manipulate the pieces). In accordance with the literature, if older adults had difficulty processing feedback and integrating it with a potential movement plan, then a change in movement performance would be observed. For Task 2, this resulted in a reduction in manual asymmetry in older adults.

This study is not without its limitations. The use of more sophisticated equipment for data collection other than a stopwatch was not possible at the time of data collection. The collection of additional kinematic variables (endpoint error, hand path) and the use of other equipment (eye-tracking devices) would allow for a more detailed examination of performance between young and older adults, leading to better insights into specific movement control strategies that change with age. Second, while every effort was taken to recruit male older adults for this study, the majority of older participants that volunteered for the study were female. An equal number of men and women would be more representative of individuals in the general population. Finally, the tasks in this study are purely behavioral in nature. Any relationships discussed between brain function and reductions manual asymmetry with age can only be inferred and referenced back to hemispheric asymmetry theories found in the literature. Thus, specific functional changes in the central nervous system with age discussed in this study cannot be fully substantiated without the use of imaging techniques such as PMRI or PET.

In summary, the results from this study revealed that the reductions in manual asymmetry were observed with an additional visuospatial component to a motor task, and these reductions in manual asymmetry were specifically observed in the older adults in Task 2. Future research is aimed at more detailed examination the impact of feedback on changes in manual asymmetry with age.

\section{References}

1. Baweja, H. S., Kwon, M., Onushko, T., Wright, D. L., Corcos, D. M., \& Christou, E. A. (2015). Processing of visual information compromises the ability of older adults to control novel fine motor tasks. Experimental Brain Research, 233, 34753488. doi:10.1007/s00221-015-4408-4.

2. Bopp, K. L., \& Verhaeghen, P. (2007). Age-related differences in control processes in verbal and visuospatial working memory: Storage, transformation, supervision, and coordination. Journal of Gerontology: Psychological Sciences, 62B(5), P239-P246.

3. Cabeza, R. (2002). Hemispheric asymmetry reduction in older adults: The HAROLD model. Psychology and Aging, 17(1), 85-100. doi:10.1037//0882-7974.17.1.85.

4. Cabeza, R., Anderson, N. D., Locantore, J. K., \& McIntosh, A. R. (2002). Aging gracefully: Compensatory brain activity in high-performing older adults. Neurolmage, 17, 1394-1402. doi:10.1006/nimg.2002.1280.

5. Cabeza, R., Daselaar, S. M., Dolcos, F., Prince, S. E., Budde, M., \& Nyberg, L. (2004). Task independent and task-specific age effects on brain activity during working memory, visual attention and episodic retrieval. Cerebral Cortex, 14, 364375. doi:10.1093/cercor/bhg133.

6. Cabeza, R., Grady, C. L., Nyberg, L., McIntosh, A. R., Tulving, E., Kapur, S., et al. (1997). Age related differences in neural activity during memory encoding and retrieval: A positron emission tomography study. Journal of Neuroscience, 17, 391-400.

7. Cansino, S., Guzzon, D., Martinelli, M., Barollo, M., \& Casco, C. (2011). Effects of aging on 
interference control in selective attention and working memory. Memory \& Cognition, 39, 14091422. doi:10.3758/s13421-011-0109-9

8. Chua, R., Pollock, B. J., Elliott, D., Swanson, L. R., \& Carnahan, H. (1995). The influence of age on manual asymmetries in movement preparation and execution. Developmental Neuropsychology, 11, 129-137.

9. Dolcos, F., Rice, H. J., \& Cabeza, R. (2002). Hemispheric asymmetry and aging: Right hemisphere decline or asymmetry reduction. Neuroscience and Biobehavioral Reviews, 26, 819825.

10. Flink, T. S., \& Iorio, A. N. (2015). An examination of change in control of the right and the left hand across the lifespan. Journal of Motor Learning and Development, 3, 11-22.

11. Francis, K. L., \& Spirduso, W. W. (2000). Age differences in the expression of manual asymmetry. Experimental Aging Research, 26, 169-180.

12. Guzzetti, S., \& Daini, R. (2014). Inter-hemispheric recruitment as a function of task complexity, age and cognitive reserve. Aging, Neuropsychology, and Cognition, 21(6), 722-745. doi:10.1080/13825585.2013.874522

13. Hale, S., Rose, N. S., Myerson, J., Strube, M. J., Sommers, M., Tye-Murray, N., \& Spehar, B. (2011). The structure of working memory abilities across the adult life span. Psychology and Aging, 26(1), 92-110. doi:10.1037a0021483

14. Hausmann, M., Kirk, I. J., \& Corballis, M. C. (2004). Influence of task complexity on manual asymmetries. Cortex, 40, 103-110.

15. Hutchinson, S., Kobayashi, M., Horkan, C. M., Pascual-Leone, A., Alexander, M. P., \& Schlaug,

16. G. (2002). Age-related differences in movement representation. NeuroImage, 17, 1720-1728. doi:10.1006/nimg.2002.1309

17. Kalisch, T., Wilimzig, C., Kleibel, N., Tegenthoff, M., \& Dinse, H. R. (2006). Age-related attenuation of dominant hand superiority. PloS ONE, 1, e90. doi:10.1371/journal.pone.0000090

18. Li, Z., Moore, A. B., Tyner, C., Hu, X. (2009). Asymmetric connectivity reduction and its relationship to "HAROLD" in aging brain. Brain Research, 1295, 149-158. doi:10.1016/j.brainres.2009.08.004

19. Mitrushina, M., Fogel, T., D'Elia, L., Uchiyama, C., \& Satz, P. (1995). Performance on motor tasks as an indication of increased behavioral asymmetry with advancing age. Neuropsychologia,33, 359364.

20. Naccarato, M., Calautti, C., Jones, P. S., Day, D. J., Carpenter, T. A., \& Baron, J.-C. (2006). Does healthy aging affect the hemispheric activation balance during paced index-to-thumb opposition task? An fMRI study. NeuroImage, 32, 1250-1256. doi:10.1016/j.neuroimage.2006.05.003

21. Oldfield, R. C. (1971). The assessment and analysis of handedness: The Edinburgh Inventory. Neuropsychologia, 9, 97-113.

22. Park, D. C., Lautenschlager, G., Hedden, T., Davidson, N. S., Smith, A. D., \& Smith, P. K. (2002). Models of visuospatial and verbal memory across the adult life span. Psychology and Aging, 17, 299-320.

23. Piefke, M., Onur, O. A., \& Fink, G. R. (2012). Aging-related changes in neural mechanisms underlying visual-spatial working memory. Neurobiology of Aging, 33, 1284-1297. doi:10.1016/j.neurobiolaging.2010.10.014.

24. Pohl, P. S., Winstein, C. J., \& Fisher, B. E. (1996). The locus of age-related movement slowing: Sensory processing in continuous goal-directed aiming. Journal of Gerontology: Psychological Science, 51B, P94-P102.

25. Provins, K. A. (1997). The specificity of motor skill and manual asymmetry: A review of the evidence and its implications. Journal of Motor Behavior, 29(2), 183-192.

26. Przybyla, A., Haaland, K. Y., Bagesteiro, L. B., \& Sainburg, R. L. (2011). Motor asymmetry reduction in older adults. Neuroscience Letters,489, 99-104. doi:10.1016/j.neulet.2010.11.074.

27. Sainburg, R. L. (2005). Handedness: Differential specializations for control of trajectory and position. Exercise and Sport Sciences Reviews, 33(4), 206-213.

28. Sainburg, R. L., \& Kalakanis, D. (2000). Differences in control of limb dynamics during dominant and nondominant arm reaching. Journal of Neurophysiology, 83, 2661-2675.

29. Seidler-Dobrin, R. D., \& Stelmach, G. E. (1998). Persistence in visual feedback control by the elderly. Experimental Brain Research, 119, 467474.

30. Teixeira, L. A. (2008). Categories of manual asymmetry and their variation with advancing age. Cortex, 44, 707-716. doi:10.1016/j.cortex.2006.10.002.

31. Temprado, J-J., Sleimen-Malkoun, R., Lemaire, P., Rey-Robert, B., Retornaz, F., \& Berton, E. (2013). Aging of sensorimotor processes: A systematic study in Fitts' task. Experimental Brain Research, 228, 105-116. doi:10.1007/s00221-013-3542-0.

32. Wang, J., D'Amato, A., Bambrough, J., Swartz, A. M., \& Miller, N. E. (2016). A positive association between active lifestyle and hemispheric lateralization for motor control and learning in older adults. Behavioural Brain Research, 314, 38-44. Retrieved from http://dx.doi.org/10.1016/j.bbr.2016.07.048 
33. Wang, J., Przybyla, A., Wuebbenhorst, K., Haaland, K. Y., \& Sainburg, R. L. (2011). Aging reduced asymmetries in interlimb transfer of visuomotor adaptation. Experimental Brain Research, 210(2), 283-290. doi:10.1007/s00221011-2631-1.

34. Wang, J., \& Sainburg, R. L. (2007). The dominant and non-dominant arms are specialized for stabilizing different features of task performance. Experimental Brain Research, 178, 565-570. doi:10.1007/s00221-007-0936-x.

35. Yan, J. H., Thomas, J. R., \& Stelmach, G. E. (1998). Aging and rapid aiming arm movement control. Experimental Aging Research, 24, 155-168.

36. Yan, J. H., Thomas, J. R., Stelmach, G. E., \& Thomas, K. T. (2000). Developmental features of rapid aiming arm movements across the lifespan. Journal of Motor Behavior, 32(2), 121-140.

37. Yeh, T-T., Cinelli, M. E., Lyons, J. L., \& Lee, T. D. (2015). Age-related changes in postural control to the demands of a precision task. Human Movement Science, 44, 134-142. Retrieved from http://dx.doi.org/10.1016/j.humov.2015.08.021. 